http://jmscr.igmpublication.org/home/ ISSN (e)-2347-176x ISSN (p) 2455-0450 crossref DOI: https://dx.doi.org/10.18535/jmscr/v7i9.77

\title{
Evaluation of the effects of Aqueous and Ethanolic Extracts of Morus mesozygia Linn. Stapf., Leaves on Lipid Profile and Renal Indices of Streptozotocin- Induced Diabetic Rats
}

\author{
Authors \\ M.T. Joshua ${ }^{* 1}$, E.O. Nwachuku ${ }^{1}$, N. Boisa ${ }^{2}$, Nsirim Nduka ${ }^{1}$ \\ ${ }^{1}$ Department of Medical Laboratory Science, Rivers State University Port Harcourt \\ ${ }^{2}$ Department of Chemistry, Rivers State University Port Harcourt \\ *Corresponding Author \\ M.T. Joshua \\ Department of Medical Laboratory Science, Rivers State University Port Harcourt
}

\begin{abstract}
Diabetes mellitus as a metabolic disorder invades with characterized hyperglycemia as well as glycosuria that if not treated properly can lead to the amputation of the arms and limbs. The increase in medi-care cost and the side effects that comes from the use of orthodox drugs has made scientists to look into phytomedicine, the use of plant herbs as an alternative for treatment modalities. Morus mesozygia Linn Stapf., was the medicinal plant chosen for this research study. The study evaluated the effects of Morus mesozygia Linn. Stapf., Leaves and Twigs on lipid profile and renal indices in Streptozotocin-Induced Diabetic Rats. A total of fifty (50) male albino rats were used for the study. Two different extracted solvents; aqueous, and ethanolic leaves extracts were used. The male albino rats for this study were induced with a single dose of $40 \mathrm{mg} / \mathrm{kg}$ b.wt, streptozotocin in $0.1 \mathrm{M}$ of citrate buffer, $\mathrm{pH} 4.5$ intraperitoneally. The diabetic male rats were those whose fasting blood glucose $(F B G)$ were from $250 \mathrm{mg} / \mathrm{dl}$ or $13 \mathrm{mmol} / \mathrm{L}$ and above. TCHOL, TG \& HDL-C were analyzed by enzymatic method, LDL-C by Frieldwald's calculation. Electrolytes $\left(\mathrm{K}^{+}, \mathrm{Na}^{+}, \mathrm{Cl}, \mathrm{HCO}_{3}^{-}\right)$were analyzed by ion-selective electrode. A significant decrease $(p<0.05)$ in TCHOL, TRIG, LDL and increased HDL levels when compared with those of the aqueous leaves and ethanolic leave extracts. The ethanolic leaves extract exhibited anti-hypercholesterolemic, anti-lipidemic activities more than the aqueous extract, this may be probably due to ethanol having a greater polarity index as well as exhibiting more components such as polyphenols, flavonoids, tannins, saponins, alkaloids. Keywords: Aqueous, Ethanolic Extracts, Morus mesozygia Linn. Stapf., Leaves, Lipid Profile, Renal Indices Streptozotocin-Induced, Diabetic Rats.
\end{abstract}

\section{Introduction}

One of the biggest diseases globally that increasingly drains the budget of the private health sector annually is diabetes mellitus. Diabetes mellitus has been acknowledged as one of the world's leading causes of disability and

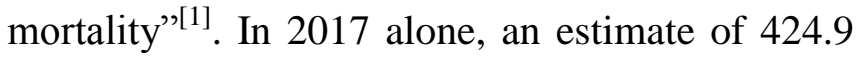
million adults worldwide as provided by the International Diabetes Federation, were sufferers of diabetes mellitus, what is alarming is that this estimate by the year 2040 is expected to rise to 776 million individuals living with diabetes. It has 
been reported that about 50 percent of these individuals do not know they have diabetes, a scourge in annual expenditure for healthcare delivery globally has risen from 727 billion USD in the year 2017 with a massive 776 million USD that is expected to be budgeted on for the year 2040, this increase is expected for treatment due to both an incidence of insulin resistance as well as the prevalence of type 2 diabetes mellitus which has been the most prevalent due to unhealthy feeding habits and also sedentary lifestyle.

According by the assessment carried out by ${ }^{[2]}$, results revealed about 40 percent of individuals with elevated blood glucose cases levels resulting into cases of Chronic Kidney Diseases as well as type two diabetic kidney disease (DKD), an equivalent of well over 29 million individuals in the United States of America.

For the purpose of proper diagnosis and assessment of Morus Mesozygia Linn. leaves in the cause of digestion and reabsorption in this research study, it is important to carry out laboratory investigations of certain electrolytes such as bicarbonates, sodium, potassium, as the kidney does this in a delicate sensory mechanism $^{[3]}$. Therefore, the aim of the study was to assess the effect of Morus Mesozygia Linn. Leaves on the lipid profile and electrolytes of streptozotocin induced diabetic rats.

\section{Materials and Methods}

\section{Animal Preparation}

All male albino rats of (150g to $200 \mathrm{~g}$ ) in weight were purchased from the University of Port Harcourt. They were used throughout the course of this research work and were made to acclimatize for 14days under standard laboratory conditions, fed with pelleted rat chow (Top Feed Finisher Mash, Nigeria) and tap water ad libitum.

The rats were fed with high fatty feeds which was commercially prepared with margarine and sucrose (see appendix for parts preparation) in combination with the pelleted chow to initiate obesity, recent studies have reported that high fatty diets give out free radicals that contribute to the impairment of beta cells hence hyperglycemia and its subsequent complications (Bahmani et al., 2014).

\section{Plant Collection and Authentication}

Morus mesozygia Linn.(family Moraceae) fresh leaves and twigs samples were collected by Dr. Oladele, A.T. in the month of July, 2018 from an abandoned, fallow- farmland at Ile -Ife, Ilesha Road, Ile-Ife, Osun State, South-Western Nigeria and was authenticated by plant botanist, Dr. Oladele A.T.at the Department of Forestry and Wildlife Management, University of Port Harcourt with the herbarium voucher number (UPFH 0125) and was submitted at the department's herbarium.

\section{Preparation of Plant Extract (Cold Maceration} Extraction Method)

The Morus mesozygia linn leaves and twigs were washed with distilled water and air dried separately for seven days and milled into fine powder with the use of a milling machine, the powdered leaves produced a total weight of $2.90 \mathrm{~kg}$, the powered twigs weighed a total of $2.45 \mathrm{~kg}$; they were stored and labelled into their different air tight containers prior to use.

Extraction of Powdered Morus mesozygia linn leaves using absolute Ethanol

Nine hundred and sixty grams (960g) of dried powdered Morus mesozygialinn leaves was put into a clean beaker, fiveliters $(5 \mathrm{~L})$ of ethanol was suspended into the beaker, this was shaken severally on a shaker,it was mixed properly and was stored for 24hours. It was macerated and filtered through a muslin cloth and again filtered out through a What Man's number one filter paper. The filtered extracts were concentrated (on low pressure) using the rotary evaporator equipment $^{[4]}$ after which they were dried on an evaporating dish at a temperature of $50^{\circ} \mathrm{C}$ to $60^{\circ} \mathrm{C}$ to a semi- solid form. A sticky semi-solid dark brownish substance was obtained. The extract was stored in a well corked universal bottle. The leaf ethanol extract yielded (18.65) g (Appendix I for 
calculation) and was kept in a $4^{\circ} \mathrm{C}$ refrigerator prior to pharmacological investigations.

The same method was used in the aqueous extraction, but instead, 5L of distilled water was used.

\section{Aqueous and Ethanolic Extract Dosage Calculation}

Based on the results from the Acute Toxicity test carried out, doses adopted for this research study that was administered orally into the rats were $200 \mathrm{mg} / \mathrm{kg}$ (low dose dose) and 400mg/kg (high) respectively. The average weights of the experimental rats in each of the groups were taken as these were used to calculate the doses of the extracts that were administered. That is, Average weight of rats in group $1=150 \mathrm{~g}$.

\section{Metformin Dosage Administration}

The metformin round tablet brand of Sandox tablet of $500 \mathrm{mg}$ was crushed and dissolved in normal saline containing $0.9 \%$ of sodium chloride (weight per volume) sodium citrate for the oral administration into the fasted diabetic rats as desired doses of $100 \mathrm{mg} / \mathrm{kg}$ used by Metformin direct calculation of animal dose from human dose.

\section{Citrate Buffer Solution Preparation}

The citrate buffer solution is a combination of citric acid salt and sodium citrate salt.

About 1.47 grams of the sodium citrate salt was measured and dissolved in $50 \mathrm{ml}$ of distilled water, this was followed by weighing 1.05 gram of citric acid salt which was dissolved in $50 \mathrm{ml}$ of distilled water. The mixtures were thoroughly stirred to enable it evenly mixed together and a $\mathrm{PH}$ meter was used to check and adjust the $\mathrm{pH}$ buffer to 4.5 (It is important to put into consideration the citric acid monohydrate of $0.1 \mathrm{M}$ solution contains $21.01 \mathrm{~g} / \mathrm{L}$. The Tri-sodium citratedehydrates of $0.1 \mathrm{M}$ solution contains $29.41 \mathrm{~g} / \mathrm{L}$ )

\section{Diabetes Induction with Streptozotocin}

After two weeks of acclimatization, the diabetes was induced in the male albino rats with streptozotozin (STZ, Sigma Chemical Company, St. Louis, Milestone). STZ was intravenously (i.v.) administered in a dose of $40 \mathrm{mg} / \mathrm{kg}$ dissolved in citrate buffer $(0.1 \mathrm{M}, \mathrm{pH} 4.5)$. Blood glucose concentrations were measured by Fine Test glucometer (Johnson \& Johnson) after 48 hours and subsequently throughout the experiment after diabetes induction and glucose concentrations exceeded $250 \mathrm{mg} / \mathrm{dl}$ or $13 \mathrm{mmol} / \mathrm{L}$ confirmed the diabetic state ${ }^{[5]}$. The diabetic male rats were picked and used for the study design.

Administration of Morus mesozygia linn. (African mulberry) for Treatment

After the rats were confirmed diabetic at above $13 \mathrm{mmol} / \mathrm{L}$, blood samples were collected from the tail artery and orbital sinus of the rats. The assay of the blood glucose levels was carried out by the glucose-oxidase principle ${ }^{[6]}$. Finetest ${ }^{\mathrm{TM}}$ test strips and Fine Test Auto Coding ${ }^{\text {TM }}$ Premium Glucometer, INFOPIA Company, Limited, Korea) was used for the determination of the blood glucose levels of the animals and the results expressed as $\mathrm{mmol} / \mathrm{L}$.

The administration of the Morus mesozygia linn. for the leaf aqueous and ethanol extracts were administered by the use of oral gavage method.

\section{Study Design}

The rats were allowed to incubate by acclimatizing for two weeks prior to the progression of the study. They were randomly separated into twenty-five (26) groups and the research study was carried out as follows:

Group One: 5 male rats were given pellet feeds and water ad libitum, this served as the 'Negative Control' group

Group Two: 5 male rats were induced intraperitoneally with a single dose of $40 \mathrm{mg} / \mathrm{kg}$ body weight of streptozotocin and were fed with pellets and water ad libitum, this served as the 'Positive Control' group

Group Three: 5 male rats were given $400 \mathrm{mg} / \mathrm{kg}$ body weight orally with aqueous leaf extract only Group Four: 5 male rats were given $400 \mathrm{mg} / \mathrm{kg}$ body weight orally with ethanolic leaf extract only Group Five: 5 male rats were induced with a single dose of $40 \mathrm{mg} / \mathrm{kg}$ body weight of streptozotocin and treated with $400 \mathrm{mg} / \mathrm{kg}$ body weight of aqueous leaf extract 
Group Six: 5 male rats were induced intraperitoneally with a single dose of $40 \mathrm{mg} / \mathrm{kg}$ body weight of streptozotocin and treated orally with $200 \mathrm{mg} / \mathrm{kg}$ body weight of aqueous leaf extract

Group Seven: 5 male rats were induced intraperitoneally with a single dose of $40 \mathrm{mg} / \mathrm{kg}$ body weight of streptozotocin and treated orally with $400 \mathrm{mg} / \mathrm{kg}$ body weight of ethanolic leaf extracts

Group Eight: 5 male rats were induced intraperitoneally with a single dose of $40 \mathrm{mg} / \mathrm{kg}$ body weight of streptozotocin and treated orally with $200 \mathrm{mg} / \mathrm{kg}$ body weight of ethanolic leaf extracts.

Group Nine: 5 male rats were induced intraperitoneally with a single dose of $40 \mathrm{mg} / \mathrm{kg}$ body weight of streptozotocin and treated orally with $100 \mathrm{mg} / \mathrm{kg}$ body weight of metformin standard drug.

Group Ten: 5 male rats were induced intraperitoneally with a single dose of $40 \mathrm{mg} / \mathrm{kg}$ body weight of streptozotocin and treated orally with $400 \mathrm{mg} / \mathrm{kgbody}$ weight of aqueous leaf extract and $100 \mathrm{mg} / \mathrm{kg}$ of metformin.

\section{Collection of Sample for Laboratory Analysis}

The rats were kept on fasting for 6 hours prior to the process of euthanasia, they were also weighed before the process started. Blood samples were collected for analysis into Lithium heparin anticoagulant bottles for biochemical test which was allowed to sit for some minutes and spun at 3,000r.p.m for 5minutes to get the serum for the analysis of lipid profile.

\section{Determination of Serum Total Cholesterol}

The method used to analyze total cholesterol was as decribed by ${ }^{[7]}$.

\section{Triglycerides Determination}

The determination of triglycerides in the samples was done by the Glycerokinase peroxidase method, a colorimetric enzymatic method described by ${ }^{[8]}$.
Determination of High Density LipoproteinCholesterol

The method used to analyze High Density Lipoprotein-Cholesterol was as decribed by ${ }^{[9]}$.

Estimation of Low Density LipoproteinCholesterol

The estimation of Low density lipoprotein (LDL) was established using the formulae proposed by [10]:

LDL - Cholesterol $(\mathrm{mg} / \mathrm{dl})=$ Total CholesterolHigh density lipoprotein- Triglycerides 5

Determination of Plasma Creatinine by Jaffe's Colorimetric Method

The method used to analyze Creatinine was as decribed by $\iota^{[11]}$.

\section{Determination of Plasma Urea}

Bertholot's Enzymatic Method was used to analyze plasma urea.

\section{Determination of Sodium by Ion-Selective Electrode}

The principle of determination of sodium was based on the selection of chromogen whose ability is based directly on its chromophore capacity as measured on its concentration and absorbance of the presence of sodium in the test sample.

\section{Determination of Bicarbonate by Ion-Selective Electrode}

Serum bicarbonates reacts with excess standard hydrochloric acid while the remaining hydrochloric acid is back titrated with standard sodium hydroxide with the use of phenol red as an indicator.

\section{Determination of Chloride by Ion-Selective Electrode}

Chloride ions react with the combination of mercurous thiocyanate to produce mercury per chlorate and thiocyanate. The presence of a red complex as a result of the formation of thiocyanate with ferric ions was formed in the presence of nitric acid.

\section{Determination of Potassium by Ion-Selective Electrode}

The concentration of potassium was measured by the inclusion of sodium tetraphenylboron in an 
exact mixture suitable to produce a colloidal suspension. The presence of the turbidity formed is such that was proportional to the concentration of the potassium in the test sample..

\section{Statistical Analysis}

Statistical evaluation was made possible with the application of Graph pad prism (version 5.0). Data generated were revealed as mean and standard deviations (Mean \pm SD) in addition to the use of ANOVA (Turkey's Multiple Comparative Test) since the comparism is within more than two group study. The level of significance was tested at $(\mathrm{p}<0.05)$.

\section{Results}

Table 4.34: Mean and Standard Deviations of Lipid Profiles of Streptozotocin Induced Diabetic Male Rats Treated Orally for 30 days with Aqueous Leaves of Morus mesozygia Linn. Stapf. Extracts compared with controls.

\begin{tabular}{|c|c|c|c|c|}
\hline & $\begin{array}{l}\mathrm{CHOL} \\
(\mathrm{mg} / \mathrm{dl})\end{array}$ & $\overline{T R I G(m g / d l)}$ & $H D L(m g / d l)$ & $L D L(m g / d l)$ \\
\hline GRP1NC & $205.78 \pm 2.56$ & $187.26 \pm 5.21$ & $182.35 \pm 19.63$ & $26.75 \pm 2.72$ \\
\hline GRP2PC & $194.36 \pm 1.19$ & $182.57 \pm 3.65$ & $101.86 \pm 10.06$ & $54.01 \pm 9.78$ \\
\hline GRP3NDALO & $198.98 \pm 4.42$ & $183.42 \pm 3.56$ & $109.9 \pm 3.40$ & $50.54 \pm 9.09$ \\
\hline GROP5DA400mg & $197.45 \pm 3.44$ & $180.66 \pm 3.41$ & $94.26 \pm 0.24$ & $66.63 \pm 2.83$ \\
\hline GRP6DA200mg & $\begin{array}{l}222.71 \pm \\
55.88\end{array}$ & $190.21 \pm 5.67$ & $180.88 \pm 32.34$ & $37.64 \pm 1.73$ \\
\hline GRP9MF100mg & $194.33 \pm 2.94$ & $180.32 \pm 1.90$ & $163.89 \pm 5.01$ & $6.90 \pm 2.89$ \\
\hline p-values & 0.3786 & 0.0047 & $<0.0001$ & $<0.0001$ \\
\hline F-values & 1.116 & 4.546 & 32.42 & 67.02 \\
\hline Tukey's Multiple Comparison Test & Summary & Summary & Summary & Summary \\
\hline $\begin{array}{l}\text { Positive control group vs Negative } \\
\text { control group }\end{array}$ & Ns & Ns & $* * *$ & $* * *$ \\
\hline Positive control group vs Group3 & Ns & Ns & $* * *$ & $* * *$ \\
\hline Positive control group vs Group5 & Ns & Ns & *** & *** \\
\hline Positive control group vs Group6 & Ns & Ns & Ns & Ns \\
\hline Positive control group vs Group9 & Ns & Ns & Ns & $* * *$ \\
\hline Negative control group vs Group3 & Ns & Ns & Ns & Ns \\
\hline Negative control group vs Group5 & Ns & Ns & Ns & $*$ \\
\hline Negative control group vs Group6 & Ns & Ns & $* * *$ & $* *$ \\
\hline Negative control group vs Group9 & Ns & Ns & $* * *$ & $* * *$ \\
\hline Group3 vs Group4 & Ns & Ns & Ns & $* *$ \\
\hline Group3 vs Group6 & Ns & Ns & $* * *$ & $*$ \\
\hline Group3 vs Group9 & Ns & Ns & $* * *$ & $* * *$ \\
\hline Group5 vs Group6 & Ns & $*$ & $* * *$ & $* * *$ \\
\hline Group5 vs Group 9 & Ns & Ns & $* * *$ & $* * *$ \\
\hline Group6 vs Group 9 & Ns & $* *$ & Ns & $* * *$ \\
\hline
\end{tabular}

Note: GRP1- Negative control, GRP2PC - Positive control, GRP 3NDALO -Non Diabetic Aqueous Leaves Orally fed with $400 \mathrm{mg}$ of Aqueous leaves extract of $(M M L S$.) extract only, GRP5- Diabetic male rats treated orally with 400mg dose of Aqueous(MMLS.), GRP 6- Diabetic male rats treated with 200mg dose of Aqueous leaves extracts of (MMLS.), GRP 9- Diabetic male rats treated with $100 \mathrm{mg}$ dose of standard Metformin drug. 


\section{JMSCR Vol||07||Issue||09||Page 445-456||September}

Table 4.37: Mean and Standard deviation of Potassium, Chloride, Sodium, Bicarbonate, Creatinine and Urea Parameters of Streptozotocin Induced Diabetic Male Rats Treated Orally with $400 \mathrm{mg} / \mathrm{kg}, 200 \mathrm{mg} / \mathrm{kg}$ for 30 days with Aqueous Leaves of Morus mesozygia Linn. Stapf. Extracts compared with 100mg/kg in Dosages of Metformin Standard with Non-Treated Controls.

\begin{tabular}{|c|c|c|c|c|c|c|}
\hline & $\mathrm{K}+(\mathrm{mmol} / \mathrm{L})$ & $C L-(\mathrm{mmol})$ & $\mathrm{Na}+(\mathrm{mmol} / \mathrm{L})$ & $\begin{array}{c}\mathrm{HCO3-} \\
(\mathrm{mmol} / \mathrm{L})\end{array}$ & $\mathrm{Cr}(\mathrm{mmol} / \mathrm{L})$ & Urea $(\mathrm{mmol} / \mathrm{L})$ \\
\hline GRP1NC & $4.34 \pm 0.71$ & $44.6 \pm 1.52$ & $143.4 \pm 3.36$ & $23.4 \pm 2.07$ & $154.4 \pm 13.03$ & $13.93 \pm 0.71$ \\
\hline GRP2PC & $5.98 \pm 0.13$ & $49.8 \pm 1.3$ & $152 \pm 2.44$ & $19.4 \pm 1.52$ & $173.8 \pm 3.34$ & $18.19 \pm 1.24$ \\
\hline GRP3NDALO & $4.20 \pm 0.76$ & $41.4 \pm 1.14$ & $137.8 \pm 5.89$ & $28.8 \pm 1.30$ & $153 \pm 23.27$ & $11.81 \pm 0.78$ \\
\hline GRP5DA400mg & $4.18 \pm 0.23$ & $42.8 \pm 1.92$ & $138.8 \pm 1.30$ & $25.2 \pm 2.48$ & $161.8 \pm 12.98$ & $16.29 \pm 1.12$ \\
\hline GRP6DA200mg & $4.38 \pm 0.23$ & $42 \pm 1.58$ & $135 \pm 3.80$ & $25 \pm 3.39$ & $137 \pm 6.16$ & $11.28 \pm 0.43$ \\
\hline GRP9DMF100mg & $3.53 \pm 0.72$ & $38.8 \pm 5.16$ & $133.4 \pm 4.44$ & $26.2 \pm 2.16$ & $166.4 \pm 10.4$ & $11.55 \pm 0.93$ \\
\hline p-values & $<0.0001$ & $<0.0001$ & $<0.0001$ & $<0.0001$ & 0.1429 & $<0.0001$ \\
\hline F-values & 13.15 & 18.32 & 10.52 & 17.01 & 1.84 & 35.15 \\
\hline $\begin{array}{l}\text { Tukey's Multiple } \\
\text { Comparison Test }\end{array}$ & Summary & Summary & Summary & Summary & Summary & Summary \\
\hline GROUP1 vs GROUP2 & $* *$ & $*$ & Ns & $*$ & Ns & $* * *$ \\
\hline GROUP1 vs Group3 & Ns & Ns & Ns & $* *$ & Ns & $*$ \\
\hline GROUP1 vs Group5 & Ns & $*$ & Ns & $* *$ & Ns & $* *$ \\
\hline GROUP1 vs Group6 & Ns & Ns & Ns & Ns & Ns & $*$ \\
\hline GROUP1 vs Group9 & Ns & $*$ & $*$ & Ns & Ns & $*$ \\
\hline GROUP2 vs Group3 & $* * *$ & $* * *$ & $* * *$ & $* * *$ & Ns & $* * *$ \\
\hline GROUP2 vs Group5 & $* * *$ & $* * *$ & $* * *$ & $* * *$ & Ns & ns \\
\hline GROUP2 vs Group6 & $* * *$ & $* *$ & $* *$ & $* * *$ & Ns & ns \\
\hline GROUP2 vs Group9 & $* * *$ & $* * *$ & $* * *$ & $* * *$ & Ns & $* * *$ \\
\hline Group3 vs Group9 & Ns & Ns & Ns & Ns & Ns & $* * *$ \\
\hline Group3 vs Group6 & Ns & Ns & Ns & Ns & Ns & $* * *$ \\
\hline Group3 vs Group9 & Ns & Ns & Ns & Ns & Ns & ns \\
\hline Group5 vs Group6 & Ns & Ns & Ns & Ns & Ns & ns \\
\hline Group5 vs Group9 & Ns & Ns & Ns & Ns & Ns & $* * *$ \\
\hline Group6 vs Group9 & Ns & Ns & Ns & Ns & Ns & $* * *$ \\
\hline
\end{tabular}

Note: GRP1- Negative control, GRP2PC - Positive control, GRP 3NDALO -Non Diabetic AqueousLeaves Orally fed with 400mg of aqueous leaves extract of $(M M L S$.) extract only,GRP5- Diabeticmale rats treated orally with 400mg dose of aqueous(MMLS.), GRP 6- Diabetic male rats treated with $200 \mathrm{mg}$ dose of Aqueous leaves extracts of (MMLS.), GRP 9- Diabetic male rats treated with 100mg dose of standard Metformin drug. $(p<0.05=$ Statistically Significant $)(p>0.05=$ Not Statistically Significant $)$

Table 4.46: Mean and Standard Deviations of Lipid Profiles of Streptozotocin Induced Diabetic Male Rats Treated Orally for 30 days with $400 \mathrm{mg} / \mathrm{kg}, 200 \mathrm{mg} / \mathrm{kg}$ in Doses of Ethanolic Leaves of Morus mesozygia Linn. Stapf., Extracts Compared with $100 \mathrm{mg} / \mathrm{kg}$ of metformin Standard and Non-Treated Controls.

\begin{tabular}{|c|c|c|c|c|}
\hline & CHOL $(\mathrm{mg} / \mathrm{dl})$ & $T R I G(m g / d l)$ & $H D L(m g / d l)$ & $L D L(m g / d l)$ \\
\hline GRP1NC & $190.78 \pm 2.56$ & $181.26 \pm 5.21$ & $182.35 \pm 19.63$ & $26.75 \pm 2.72$ \\
\hline GRP2PC & $194.36 \pm 1.19$ & $182.57 \pm 3.65$ & $101.86 \pm 10.06$ & $54.01 \pm 9.78$ \\
\hline GRP5DET400mg & $190.37 \pm 4.56$ & $141.64 \pm 44.5$ & $110.8 \pm 7.25$ & $59.05 \pm 16.1$ \\
\hline GRP7DET400mg & $197.36 \pm 1.97$ & $154.44 \pm 6.30$ & $202.98 \pm 26.04$ & $21.95 \pm 9.92$ \\
\hline GRP8DET200mg & $192.31 \pm 0.93$ & $184.34 \pm 1.49$ & $170.99 \pm 46.45$ & $62.18 \pm 12.95$ \\
\hline GRP9DMF100mg & $194.33 \pm 2.94$ & $180.32 \pm 1.90$ & $163.89 \pm 5.01$ & $6.90 \pm 2.89$ \\
\hline p-values & $<0.0001$ & 0.2693 & $<0.0001$ & $<0.0001$ \\
\hline F-values & 21.03 & 1.373 & 14.4 & 24.81 \\
\hline Tukey's Multiple Comparison Test & Summary & Summary & Summary & Summary \\
\hline Group 1 vs Group 2 & $*$ & $\mathrm{Ns}$ & $* * *$ & $* *$ \\
\hline Group 1 vs Group 5 & $* * *$ & Ns & *** & $* * *$ \\
\hline Group 1 vs Group 7 & $* * *$ & Ns & Ns & Ns \\
\hline Group 1 vs Group 8 & $* * *$ & Ns & Ns & $* * *$ \\
\hline Group 1 vs Group 9 & $* * *$ & Ns & Ns & Ns \\
\hline
\end{tabular}




\begin{tabular}{|c|c|c|c|c|}
\hline Group 2 vs Group 4 & Ns & Ns & Ns & Ns \\
\hline Group 2 vs Group 7 & Ns & Ns & $* * *$ & $* * *$ \\
\hline Group 2 vs Group 8 & Ns & Ns & ** & Ns \\
\hline Group 2 vs Group 9 & Ns & Ns & $* *$ & $* * *$ \\
\hline Group 4 vs Group 7 & $* *$ & Ns & $* * *$ & *** \\
\hline Group 4 vs Group 8 & Ns & Ns & ** & Ns \\
\hline Group 4 vs Group 9 & Ns & Ns & * & **** \\
\hline Group 7 vs Group 8 & Ns & Ns & Ns & $* * *$ \\
\hline Group 7 vs Group 9 & Ns & Ns & Ns & Ns \\
\hline Group 8 vs Group 9 & Ns & Ns & Ns & **** \\
\hline
\end{tabular}

Note: GRP1- Negative control, GRP2PC - Positive control, GRP 5NDMETHLO -Non Diabetic Ethanolic Leaves Orally fed with 400mg of ethanolic leaves extract of $(M M L S$.) extract only,GRP7- Diabeticmale rats treated orally with 400mg dose of ethanolic(MMLS.), GRP 8- Diabetic male rats treated with $200 \mathrm{mg}$ dose of ethanolic leaves extracts of (MMLS.), GRP 9- Diabetic male rats treated with 100mg dose of standard Metformin drug. $(\mathrm{p}<0.05=$ Statistically Significant $)(\mathrm{p}>0.05=$ Not Statistically Significant $)$

Table4.49: Mean and Standard deviation of Potassium, Chloride, Sodium, Bicarbonate, Creatinine and Urea Parameters of Streptozotocin Induced Diabetic Male Rats Treated Orally for 30 days with 400mg/kg, $200 \mathrm{mg} / \mathrm{kg}$ in Doses of Ethanolic Leaves of Morus mesozygia Linn. Stapf. Extracts compared with NonTreated Controls.

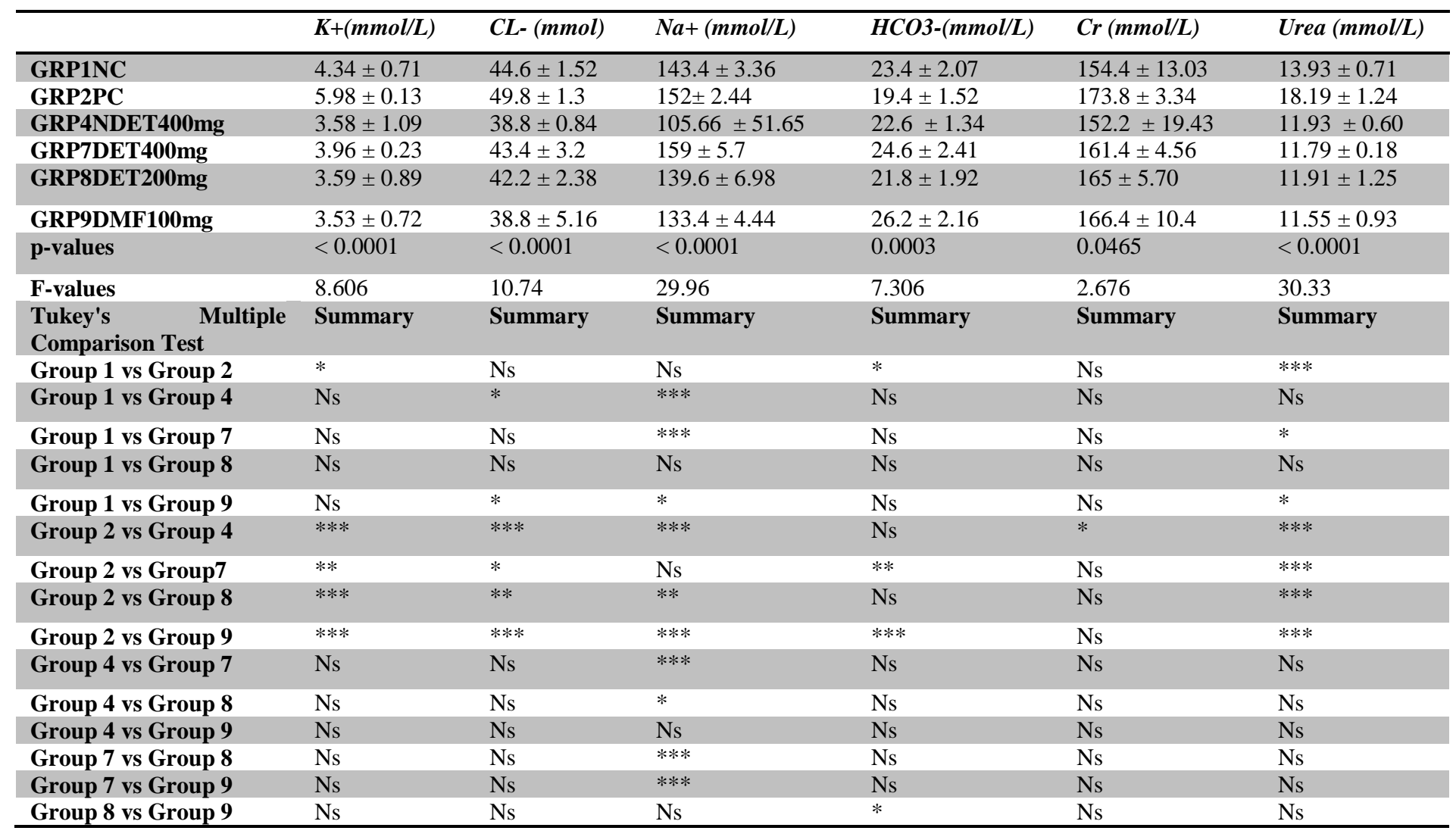

Note: GRP1- Negative control, GRP2PC - Positive control, GRP4NDMETHLO -Non Diabetic MethanolLeaves Orally fed with 400mg of ethanolic leaves extract of $(M M L S$.) extract only,GRP7- Diabeticmale rats treated orally with $400 \mathrm{mg}$ dose ofethanolic (MMLS.), GRP 8- Diabetic male rats treated with $200 \mathrm{mg}$ dose of ethanolic leaves extracts of (MMLS.), GRP 9- Diabetic male rats treated with 100mg dose of standard Metformin drug. $(\mathrm{p}<0.05=$ Statistically Significant $)(\mathrm{p}>0.05=$ Not Statistically Significant $)$ 


\section{JMSCR Vol||07||Issue||09||Page 445-456||September}

Table 4.76: Mean and Standard deviations of Lipid Parameters of Streptozotocin Induced Diabetic Male Rats Treated orally for 60 days with $400 \mathrm{mg} / \mathrm{kg}$ Aqueous Leaves Extracts, 200mg/kg Aqueous Levaes Extracts of Morus mesozygia Linn. Stapf., in Comparism with $100 \mathrm{mg} / \mathrm{kg}$ of Metformin Drug compared with controls.

\begin{tabular}{|c|c|c|c|c|}
\hline & CHOL (mg/dl) & TRIG (mg/dl) & $H D L(m g / d l)$ & $L D L(m g / d l)$ \\
\hline$\overline{\text { GROUP1NC }}$ & $199.84 \pm 0.96$ & $186.77 \pm 9.44$ & $151.13 \pm 33.67$ & $24.76 \pm 3.46$ \\
\hline GROUP 2PC & $190.73 \pm 9.71$ & $181.36 \pm 8.04$ & $127.87 \pm 26.91$ & $56.52 \pm 11.06$ \\
\hline GROUP 3NDAL400mg/kg & $182.07 \pm 3.13$ & $182.08 \pm 3.06$ & $103.28 \pm 3.40$ & $49.72 \pm 9.81$ \\
\hline GROUP 5DAL400mg/kg & $143.85 \pm 26.59$ & $158.66 \pm 4.71$ & $73.46 \pm 11.19$ & $51.21 \pm 5.22$ \\
\hline GROUP 6DAL200mg/kg & $161.86 \pm 7.24$ & $151.21 \pm 6.42$ & $146.40 \pm 7.13$ & $28.12 \pm 1.72$ \\
\hline GROUP 9DMF100mg/kg & $196.30 \pm 3.84$ & $162.11 \pm 40.72$ & $150.79 \pm 31.22$ & $16.89 \pm 2.89$ \\
\hline p-values & $<0.0001$ & 0.0162 & 0.0004 & $<0.0001$ \\
\hline F-values & 16.43 & 3.498 & 6.815 & 30.58 \\
\hline Tukey's Multiple Comparison Test & Summary & Summary & Summary & Summary \\
\hline Group 1 vs Group 2 & Ns & Ns & Ns & *** \\
\hline Group 1 vs Group 3 & Ns & Ns & Ns & **** \\
\hline Group 1 vs Group 5 & $* * *$ & Ns & $* *$ & $* * *$ \\
\hline Group 1 vs Group 6 & $* * *$ & $*$ & Ns & Ns \\
\hline Group 1 vs Group 9 & Ns & Ns & Ns & Ns \\
\hline Group 2 vs Group 3 & Ns & Ns & Ns & Ns \\
\hline Group 2 vs Group 5 & $* * *$ & Ns & $*$ & Ns \\
\hline Group 2 vs Group 6 & $*$ & Ns & Ns & $* * *$ \\
\hline Group 2 vs Group 9 & Ns & Ns & Ns & $* * *$ \\
\hline Group 3 vs Group 5 & $* * *$ & Ns & Ns & Ns \\
\hline Group 3 vs Group 6 & Ns & Ns & Ns & *** \\
\hline Group 3 vs Group 9 & Ns & Ns & Ns & $* * *$ \\
\hline Group 5 vs Group 6 & Ns & Ns & $* *$ & $* * *$ \\
\hline Group 5 vs Group 9 & $* * *$ & Ns & $* *$ & $* * *$ \\
\hline Group 6 vs Group 9 & $* *$ & Ns & Ns & $\mathrm{Ns}$ \\
\hline
\end{tabular}

Note: NC(Negative Control), PC (Positive control), NDAL400mg/kg (Non-Diabetic given orally 400mg/kg of Aqueous leaves), DAL400mg/kg

(Diabetic rats treated with Aqueous leaf $400 \mathrm{mg} / \mathrm{kg}$ ), DAL200mg $/ \mathrm{kg}$ (Diabetic rats treated orally with $200 \mathrm{mg} / \mathrm{kg}$ ) $(\mathrm{p}<0.05=\mathrm{signific}$ ant)

Table 4.79: Potassium, Chloride, Sodium, Bicarbonate, Creatinine, and Urea Parameters of Streptozotocin Induced Diabetic Male Rats Treated orally for 60 days with $400 \mathrm{mg} / \mathrm{kg}$ of Aqueous Leaves and $200 \mathrm{mg} / \mathrm{kg}$ Extracts of Morus mesozygia Linn. Stapf., in Comparism with $100 \mathrm{mg} / \mathrm{kg}$ of Metformin Drug compared with controls.

\begin{tabular}{|c|c|c|c|c|c|c|}
\hline & $\mathrm{K}+(\mathrm{mmol} / \mathrm{L})$ & $C L-(\mathrm{mmol})$ & $\mathrm{Na}+(\mathrm{mmol} / \mathrm{L})$ & $\begin{array}{c}\mathrm{HCO3-} \\
(\mathrm{mmol} / \mathrm{L})\end{array}$ & $\mathrm{Cr}(\mathrm{mmol} / \mathrm{L})$ & Urea $(\mathrm{mmol} / \mathrm{L})$ \\
\hline GROUP1NC & $3.88 \pm 0.67$ & $42.8 \pm 1.92$ & $140.4 \pm 2.30$ & $21.4 \pm 2.07$ & $146.8 \pm 7.85$ & $13.33 \pm 0.98$ \\
\hline GROUP 2PC & $5.94 \pm 0.15$ & $49.4 \pm 1.14$ & $146.6 \pm 5.89$ & $19.6 \pm 1.67$ & $143 \pm 6.16$ & $16.28 \pm 2.23$ \\
\hline GROUP 3NDAL400mg & $3.97 \pm 0.65$ & $40.4 \pm 1.14$ & $142 \pm 16.01$ & $27.2 \pm 1.48$ & $150.8 \pm 24.10$ & $11.54 \pm 1.12$ \\
\hline GROUP 5DAL400mg & $3.5 \pm 0.36$ & $38.8 \pm 1.30$ & $136.2 \pm 7.19$ & $28.6 \pm 1.67$ & $166.8 \pm 5.40$ & $17.05 \pm 0.30$ \\
\hline GROUP 6DAL 200mg & $4.1 \pm 0.15$ & $41.2 \pm 2.17$ & $139.4 \pm 0.89$ & $25.2 \pm 2.48$ & $161.8 \pm 12.98$ & $17.00 \pm 0.46$ \\
\hline GROUP9DMF100mg & $3.53 \pm 0.71$ & $38.8 \pm 5.16$ & $133.4 \pm 4.44$ & $26.2 \pm 2.16$ & $166.4 \pm 10.43$ & $11.55 \pm 0.93$ \\
\hline p-values & $<0.0001$ & $<0.0001$ & 0.1696 & $<0.0001$ & 0.0215 & $<0.0001$ \\
\hline F-values & 15.61 & 12.03 & 1.715 & 15.88 & 3.275 & 24.75 \\
\hline $\begin{array}{ll}\text { Tukey's } & \text { Multiple } \\
\text { Comparison Test } & \end{array}$ & Summary & Summary & Summary & Summary & Summary & Summary \\
\hline Group 1 vs Group 2 & $* * *$ & $* *$ & Ns & Ns & Ns & ** \\
\hline Group 1 vs Group 3 & Ns & Ns & Ns & $* *$ & Ns & Ns \\
\hline Group 1 vs Group 5 & Ns & Ns & Ns & $* * *$ & Ns & $* * *$ \\
\hline Group 1 vs Group 6 & Ns & Ns & Ns & Ns & Ns & $* * *$ \\
\hline Group 1 vs Group 9 & Ns & Ns & Ns & $* *$ & Ns & Ns \\
\hline Group 2 vs Group 3 & $* * *$ & $* * *$ & Ns & $* * *$ & Ns & $* * *$ \\
\hline Group 2 vs Group 5 & $* * *$ & $* * *$ & Ns & $* * *$ & Ns & Ns \\
\hline Group 2 vs Group6 & $* * *$ & $* * *$ & Ns & $* *$ & Ns & Ns \\
\hline Group 2 vs Group 9 & $* * *$ & $* * *$ & Ns & $* * *$ & Ns & $* * *$ \\
\hline Group 3 vs Group 5 & Ns & Ns & Ns & Ns & Ns & $* * *$ \\
\hline Group 3 vs Group 6 & Ns & Ns & Ns & Ns & Ns & $* * *$ \\
\hline Group 3 vs Group 9 & Ns & Ns & Ns & Ns & Ns & Ns \\
\hline Group 5 vs Group 6 & Ns & Ns & Ns & Ns & Ns & Ns \\
\hline Group 5 vs Group 9 & Ns & Ns & Ns & Ns & Ns & $* * *$ \\
\hline Group 6 vs Group 9 & Ns & Ns & Ns & Ns & Ns & $* * *$ \\
\hline
\end{tabular}

Note: NC(Negative Control), PC (Positive control), NDAL400mg/kg (Non-Diabetic given orally 400mg/kg of Aqueous leaves), DAL400mg/kg (Diabetic rats treated with Aqueous leaf $400 \mathrm{mg} / \mathrm{kg}$ ), DAL200mg $/ \mathrm{kg}$ (Diabetic rats treated orally with $200 \mathrm{mg} / \mathrm{kg}$ ) $(\mathrm{p}<0.05=\mathrm{significant}$ ) 


\section{JMSCR Vol||07||Issue||09||Page 445-456||September}

Table 4.88: Mean and Standard Deviations of Lipid Parameters of Streptozotocin Induced Diabetic Male Rats Treated orally for 60 days with $400 \mathrm{mg} / \mathrm{kg}$ Ethanolic Leaves Extracts, $200 \mathrm{mg} / \mathrm{kg}$ Dose of Ethanolic Levaes Extracts of Morus mesozygia Linn. Stapf., in Comparism with $100 \mathrm{mg} / \mathrm{kg}$ of Metformin Drug compared with Non-Treated controls.

\begin{tabular}{|c|c|c|c|c|}
\hline & CHOL $(\mathrm{mg} / \mathrm{dl})$ & TRIG (mg/dl) & $H D L(m g / d l)$ & $L D L(m g / d l)$ \\
\hline GRP1NC & $199.84 \pm 0.96$ & $186.77 \pm 9.44$ & $151.13 \pm 33.67$ & $24.76 \pm 3.46$ \\
\hline GRP2PC & $190.73 \pm 9.71$ & $181.36 \pm 8.04$ & $127.87 \pm 26.91$ & $56.52 \pm 11.06$ \\
\hline GRP4NDET400mg & $186.73 \pm 6.66$ & $140.76 \pm 44.54$ & $103.98 \pm 5.95$ & $56.58 \pm 15.90$ \\
\hline GRP7DET400mg & $142.36 \pm 36.47$ & $97.04 \pm 21.23$ & $153.92 \pm 13.26$ & $14.09 \pm 6.26$ \\
\hline GRP8DET200mg & $172.31 \pm 33.41$ & $154.34 \pm 36.75$ & $165.18 \pm 48.65$ & $55.58 \pm 10.51$ \\
\hline GROUP 9 & $196.30 \pm 3.84$ & $162.11 \pm 40.72$ & $150.79 \pm 31.22$ & $16.89 \pm 2.89$ \\
\hline p-values & 0.0002 & 0.0078 & 0.1253 & 0.0001 \\
\hline F-values & 5.267 & 4.102 & 1.937 & 23.72 \\
\hline Tukey's Multiple Comparison Test & Summary & Summary & Summary & Summary \\
\hline Group 1 vs Group 2 & Ns & Ns & Ns & *** \\
\hline Group 1 vs Group 4 & Ns & Ns & Ns & *** \\
\hline Group 1 vs Group 7 & $* *$ & $* *$ & Ns & Ns \\
\hline Group 1 vs Group 8 & Ns & Ns & Ns & $* * *$ \\
\hline Group 1 vs Group 9 & Ns & Ns & Ns & Ns \\
\hline Group 2 vs Group 4 & Ns & Ns & Ns & Ns \\
\hline Group 2 vs Group 7 & $*$ & $*$ & Ns & $* * *$ \\
\hline Group 2 vs Group 8 & Ns & Ns & Ns & Ns \\
\hline Group 2 vs Group 9 & Ns & Ns & Ns & $* * *$ \\
\hline Group 4 vs Group 7 & $*$ & Ns & Ns & $* * *$ \\
\hline Group 4 vs Group 8 & Ns & Ns & Ns & Ns \\
\hline Group 4 vs Group 9 & Ns & Ns & Ns & $* * *$ \\
\hline Group 7 vs Group 8 & Ns & Ns & Ns & $* * *$ \\
\hline Group 7 vs Group 9 & $* *$ & Ns & Ns & Ns \\
\hline Group 8 vs Group 9 & Ns & Ns & Ns & $* * *$ \\
\hline
\end{tabular}

Note: NC-Negative Control, PC-Positive Control, NDET400mg-Non-Diabetic fed orally with ethanolic extracts of MMLS., DET400mg-Diabtic fed orally with $400 \mathrm{mg} / \mathrm{kg}$ dose of ethanolic extracts, DET200mg-Diabetic treated with $200 \mathrm{mg} / \mathrm{kg}$ of ethanolic leaves extracts of $M M L S$., DMF100mg-Diabetic treated with $100 \mathrm{mg} / \mathrm{kg}$ of Metformin Standard drug. ( $\mathrm{p}<0.05)$ - Statistically significant)

Table 4.91: Potassium, Chloride, Sodium, Bicarbonate, Creatinine, and Urea Parameters of Streptozotocin Induced Diabetic Male Rats Treated orally for 60 days with $400 \mathrm{mg} / \mathrm{kg}$ of Ethanolic Leaves and $200 \mathrm{mg} / \mathrm{kg}$ Doses of Ethanolic Leaves Extracts of Morus mesozygia Linn. Stapf., in Comparism with 100mg/kg of Metformin Drug Compared with Non-Treated Controls.

\begin{tabular}{|c|c|c|c|c|c|c|}
\hline & $K+(\mathrm{mmol} / \mathrm{L})$ & $C L-(\mathrm{mmol})$ & $\mathrm{Na}+(\mathrm{mmol} / \mathrm{L})$ & $\begin{array}{c}\mathrm{HCO3-} \\
(\mathrm{mmol} / \mathrm{L})\end{array}$ & $\mathrm{Cr}(\mathrm{mmol} / \mathrm{L})$ & Urea $(\mathrm{mmol} / \mathrm{L})$ \\
\hline GROUP1NC & $3.88 \pm 0.67$ & $42.8 \pm 1.92$ & $140.4 \pm 2.30$ & $21.4 \pm 2.07$ & $146.8 \pm 7.85$ & $13.33 \pm 0.98$ \\
\hline GROUP 2PC & $5.94 \pm 0.15$ & $49.4 \pm 1.14$ & $146.6 \pm 5.89$ & $19.6 \pm 1.67$ & $143 \pm 6.16$ & $16.28 \pm 2.23$ \\
\hline GROUP4NDETL400mg & $2.81 \pm 0.79$ & $37.4 \pm 1.94$ & $124.4 \pm 3.84$ & $20.4 \pm 1.67$ & $138.6 \pm 25.38$ & $11.7 \pm 0.63$ \\
\hline GROUP 7DETL400mg & $3.96 \pm 0.23$ & $42.4 \pm 1.94$ & $159 \pm 5.70$ & $24.6 \pm 2.40$ & $159 \pm 5.83$ & $11.79 \pm 0.18$ \\
\hline GROUP 8DETL200mg & $3.59 \pm 0.89$ & $41 \pm 1.22$ & $139.6 \pm 6.98$ & $22.6 \pm 2.70$ & $165 \pm 5.70$ & $11.91 \pm 1.25$ \\
\hline GROUP 9DMF100mg & $3.53 \pm 0.71$ & $38.8 \pm 5.16$ & $133.4 \pm 4.44$ & $26.2 \pm 2.16$ & $166.4 \pm 10.43$ & $11.55 \pm 0.93$ \\
\hline p-values & 0.0001 & 0.0001 & 0.0001 & 0.0001 & 0.0004 & 0.0001 \\
\hline F-values & 13.44 & 12.93 & 26.53 & 6.96 & 4.606 & 11.48 \\
\hline $\begin{array}{l}\text { Tukey's Multiple } \\
\text { Comparison Test }\end{array}$ & Summary & Summary & Summary & Summary & Summary & Summary \\
\hline Group 1 vs Group 2 & $* * *$ & $* *$ & Ns & Ns & Ns & $* *$ \\
\hline Group 1 vs Group 4 & Ns & $*$ & $* * *$ & Ns & Ns & ns \\
\hline Group 1 vs Group 7 & Ns & Ns & $* * *$ & Ns & Ns & ns \\
\hline Group 1 vs Group 8 & Ns & Ns & Ns & Ns & Ns & ns \\
\hline Group 1 vs Group 9 & Ns & Ns & Ns & $*$ & Ns & ns \\
\hline Group 2 vs Group 4 & $* * *$ & $* * *$ & $* * *$ & Ns & Ns & $* * *$ \\
\hline Group 2 vs Group 7 & $* * *$ & $* *$ & $* *$ & $*$ & Ns & $* * *$ \\
\hline Group 2 vs Group 8 & $* * *$ & $* * *$ & Ns & Ns & Ns & $* * *$ \\
\hline Group 2 vs Group 9 & $* * *$ & $* * *$ & $* *$ & $* * *$ & Ns & $* * *$ \\
\hline Group 4 vs Group 7 & Ns & Ns & $* * *$ & Ns & Ns & ns \\
\hline Group 4 vs Group 8 & Ns & Ns & $* *$ & Ns & $*$ & ns \\
\hline Group 4 vs Group 9 & Ns & Ns & Ns & $* *$ & $*$ & ns \\
\hline Group 7 vs Group 8 & Ns & Ns & $* * *$ & Ns & Ns & ns \\
\hline Group 7 vs Group 9 & Ns & Ns & $* * *$ & Ns & Ns & ns \\
\hline Group 8 vs Group 9 & Ns & Ns & Ns & Ns & Ns & ns \\
\hline
\end{tabular}

Note:NC-Negative Control, PC-Positive Control, NDET400mg-Non-Diabetic fed orally with ethanolic extracts of MMLS., DET400mg-Diabtic fed orally with $400 \mathrm{mg} / \mathrm{kg}$ dose of ethanolic extracts, DET $200 \mathrm{mg}$-Diabetic treated with $200 \mathrm{mg} / \mathrm{kg}$ of ethanolic leaves extracts of $M M L S$., DMF100mg-Diabetic treated with 100mg/kg of Metformin Standard drug. $(\mathrm{p}<0.05)$ - Statistically significant) 


\section{Discussion}

The significant increase in High density cholesterol (HDL-C), that was observed when the diabetic male albino rats that were treated orally with $200 \mathrm{mg} / \mathrm{kg}$ aqueous leaves extracts of $M M L S$., showed the antihyperlipidemic activities, the dosage of this extract has when compared to the HDL-C of the male diabetic albino in the group treated with $400 \mathrm{mg} / \mathrm{kg}$ body weight of aqueous leaves extract of $M M L S$., also when compared to the HDL concentration of the diabetic rats treated with $100 \mathrm{mg} / \mathrm{kg}$ of metformin standard drug (Table 4.1); this might be due to the very high phytochemical presence of flavonoids. This was in agreement with the work done by ${ }^{[12]}$ who analysed the effect of TCHOL and LDL in rats fed orally with aqueous leaves of Morus alba Linn. and had results that revealed increased concentrations HDL and also reported in addition that these results may have caused certain stimulated actions in the innate and acquired functionings of the immunity of the cell against certain attacks of killer cells that can cause cardiovascular events.

The non-significant concentrations (Table 4.2) in $\mathrm{K}^{+}, \mathrm{Na}^{+}, \mathrm{HCO}_{3}{ }^{-}$, and decreases in Creatinine $(\mathrm{Cr})$, Chloride $\left(\mathrm{Cl}^{-}\right)$and Urea concentrations of the induced diabetic male rats treated orally with $400 \mathrm{mg} / \mathrm{kg}$ dose of aqueous leaves extracts of $M M L S$., show certain nephron-protective as well as anti-nephrotoxicity ${ }^{[13]}$ as compared to the increased levels of the afore- mentioned kidney parameters of in the diabetic rats treated orally with $200 \mathrm{mg} / \mathrm{kg}$ dose of aqueous leaves extracts of $M M L S$. This could be as result of the presence flavonoids. ${ }^{[14]}$ When compared with the concentrations in $\mathrm{K}^{+}, \mathrm{Na}^{+}, \mathrm{HCO}_{3}{ }^{-}$, Creatinine $(\mathrm{Cr})$, Chloride $\left(\mathrm{Cl}^{-}\right)$and Urea of the induced diabetic albino male rats treated orally with $200 \mathrm{mg} / \mathrm{kg}$ of aqueous leaves extracts of $M M L S$. As reports have made emphases that increase in $\mathrm{K}^{+}, \mathrm{Na}^{+}, \mathrm{HCO}_{3}{ }^{-}$, Creatinine $(\mathrm{Cr})$, Chloride $\left(\mathrm{Cl}^{-}\right)$and Urea concentrations may result into diabetic nephropathies which are implicated in end-stage renal diseases that results in high mortality rate ${ }^{[15]}$. This work was similar with that carried out by ${ }^{[15]}$, they reported a decrease in creatinine when Morus alba Linn fruit juice extracts was administered orally in amino glycosides induced renal damaged rats, they also reported that the decrease could be due to the polyphenolic contents of flavones isolated abundantly from flavonoids and that these flavones served as diuretics that have protective effects on the kidneys.

Table 4.3 revealed a decrease in total cholesterol (TCHOL), triglyceride (TG) and low density lipoprotein cholesterol (LDL-C) as well as an increased concentration in high density lipoprotein (HDL-C) in the diabetic male rats treated with $400 \mathrm{mg} / \mathrm{kg}$ of ethanolic leaves of $M M L S$ when compared with the lipid profilings of the diabetic rats treated with $200 \mathrm{mg} / \mathrm{kg}$ of ethanolic leaves extracts and those of the diabetic group treated with $100 \mathrm{mg} / \mathrm{kg}$ of metformin standard drug and those of the non-treated group. This lipid lowering capacity exhibited by the dosage of $400 \mathrm{mg} / \mathrm{kg}$ of ethanolic leaves extracts in diabetic rats may be due to the presence of the phytochemicals present which included: Saponins, Flavonoids, Tannins and Alkaloids. The cholesterols could have been decreased by the phytochemical presence of Saponins which has been reported to show certain anti-hypercholesterolemic activity it may exhibit by its ability in mechanism by the attachment to the receptors of perixosomes proliferator-activated sites that are responsible for stimulating the acceptance, usage and breakdown of fatty acids by up-regulation of genes involved in the fatty acid transport. This was similar to the work done by ${ }^{[16]}$ that induced rats with alloxan and treated them with $400 \mathrm{mg} / \mathrm{kg}$ in dosage of ethanolic Corchorus olitorius extracts for a period close to a month and observed a decrease in TCHOL, TG, LDL-C and an increase in concentration of HDL-C in the diabetic when compared with to the untreated group.

The result from Table 4.4 showed a decrease in concentration of creatinine and urea, a significant increase in $\mathrm{K}+$ and $\mathrm{HCO}^{-}$and the non-significant differences of the diabetic rats in Group 7 treated with $400 \mathrm{mg} / \mathrm{kg}$ of ethanolic leaves of $M M L S$, 
when compared to the significant decrease of $\mathrm{K}+$ and $\mathrm{HCO} 3$ - and the significant increase of creatinine and urea of the diabetic rats treated orally for 30 days with $200 \mathrm{mg} / \mathrm{kg}$ of ethanolic leaves extract may be due to the possibly protective effect the $400 \mathrm{mg} / \mathrm{kg}$ dosage of ethanolic leaves extracts has over the kidneys, this The results of significant decrease in LDL, nonsignificant TCHOL, TRIG and increased HDL levels as exhibited by the diabetic rats' treated for 60 days with $200 \mathrm{mg} / \mathrm{kg}$ of aqueous leaves on Table 4.5 is indicative of the antilipidemic effect of the dosage of this extracts on the endothelial lining and functioning of the arterial wall when compared with the increased LDL, and decreased HDL that the diabetic rats treated with $400 \mathrm{mg} / \mathrm{kg}$ of aqueous leaves extracts of $M M L S$ exhibited. This antioxidative effcts may be due to the presence of flavonoids. This was similar to the work of ${ }^{[16]}$ who observed decrease in LDL and an increase of HDL in Morus alba against the invasion of macrophages.

The significant decreased urea and creatinine and the non-significant differences of $\mathrm{K}+, \mathrm{Na}+, \mathrm{Cl}-$, $\mathrm{HCO} 3$ - exhibited by the diabetic rats treated with $200 \mathrm{mg} / \mathrm{kg}$ of aqueous leaves extracts of $M M L S$ on Table 4.6 indicated that the glomerular filtration rate of the kidney with the reabsorption of the electrolytes balanced for the elimination of toxic wastes when compared with the increased urea and creatinine of the diabetic rats treated with $400 \mathrm{mg} / \mathrm{kg}$ dosage of aqueous leaves extracts. This nephrotoxic protection of the $200 \mathrm{mg} / \mathrm{kg}$ dosage may be due to the presence of flavonoids. This concurs with the work of ${ }^{[17]}$ who reported decrease in urea and creatinine as a result of the extracts from the fruits of Morus alba L. that served as a form of diuretics on renal functioning and clearance.

Table 4.7 revealed a significant decrease of TCHOL, TRIG, LDL and an increase in HDL levels of the diabetic rats treated for 60 days with $400 \mathrm{mg} / \mathrm{kg}$ of ethanolic leaves. This is indicative of antihypercholesteromic effect as well as antilipidemic effect this dose has in lowering cholesterol and transporting it easily into cell membranes, as a result of the increased HDL compared with that of the diabetic rats treated with $200 \mathrm{mg} / \mathrm{kg}$. This antihypercholesteromic effect exhibited may be due to saponins. Saponins may have acted in an action mechanism of attaching its perixosome proliferator recptors on lipid sites thereby regulating the upward movement of glucose into the islets cells. This work did not agree with that of ${ }^{[18]}$ that investigated the impart of $400 \mathrm{mg} / \mathrm{kg}$ of ethanolic extracts of Corchorus olitorius in diabetic alloxan induced rats and observed decreased TCHOL, TRIG, LDL within a period less than 30 days.

The significant decrease of urea and creatinine is indicative of renal protective effect of the diabetic rats treated with $200 \mathrm{mg} / \mathrm{kg}$ ethanolic leaves of $M M L S$ on Table 4.8 when compared with increased urea and creatinine of the diabetic rats treated with $400 \mathrm{mg} / \mathrm{kg}$ of ethanolic leaves. This renal protective effect of the $200 \mathrm{mg} / \mathrm{kg}$ ethanolic leave extract may be due to the presence of tannins. This agreed with the work done by ${ }^{[18]}$.

\section{Conclusion}

The combinatorial therapy of the aqueous and metformin standard drug at $400 \mathrm{mg} / \mathrm{kg}$ body weight showed a more antidiabetic potential when compared to that of metformin drug alone. The ethanolic leaves extract exhibited antihypercholesterolemic, anti-lipidemic activities more than the aqueous extract, this may be probably due to ethanol having a greater polarity index as well as exhibiting more components such as polyphenols, flavonoids,, tannins, saponins, alkaloids.

\section{References}

1. Marinho, H.S.,Real, C.,Cyrne,L.,Soares.H. \& Antlines, F. (2014). Hydrogen peroxide sensing, signaling and regulation of transcription factors. Redox Biology, 2,535562.

2. Bailey, R.A., Wang, Y., Zhu, V.\& Rupnow, M.F. (2014). Chronic kidney disease in U.S. 
adults with type 2 diabetes: An updated national estimate of prevalence based on Kidney Disease: Improving Global Outcomes (KDIGO) staging. British Medical College of Research Notes. 7,415

3. Pluznick, J.L. \& Caplan,M.J. (2015). Chemical and physical sensors in the regulation. Clinical Journal of American Society of Nephrology,10(9), 1625-1635.

4. Gulcin, M. (2005). The antioxidant and radical scavenging activities of black pepper (Pipernigrum) Seeds. International Journal of Food and Science Nutrition. 56,401-499.

5. Damasceno, A., Bongani, M., Mayosi, D. \& Mayosi, D. (2012). The causes, treatment and outcome of acute heart failure in 1006 africans from 9 counties. Results of the SubSaharan Africa survey of heart failure. Archives of Internal Medicine, 172(18),13861394.

6. Beach, E.F.\& Turner,J.J. (1958). An Enzymatic Method for Glucose Determination in Body Fluids. Clinical Chemistry, 4,462-475.

7. Allain, C.C., Poon, L.S., Chan, C.S., Richmond, W. \& Fu, P.C. (1974). Enzymatic determination of total serum cholesterol. Clinical Chemistry, 20(4),470-475.

8. Tiez,N. W. (1990).A Clinical Guide to Laboratory Tests ( $2^{\text {nd }}$ Ed) Philadelphia: W.B. Saunders.

9. Burstein, M., Scholnick, H.R. \& Morfin, R.(1970). Rapid method for the isolation of lipoprotein from serum by precipitation with polyanions. Scadinavian Journal of Clinical andLaboratory Investigation, 40,583-595.

10. Friedwald, W.T., Levy, R.I.\& Fredrickson, D.S. (1972). Estimation of the concentration of low-density lipoprotein cholesterol in plasma without the use of the preparative ultracentrifugation. Journal of Clinical Chemistry, 18,499-502.

11. Jaffe, M. (1886). Ueber den Niederschlag Welchen pikrinsaure in normalen Harn erzeugt and ubereine neue reaction des kreatinins, Hoppe-Seyler's Zeitschrift fur PhysiologischeChemie, 10, 391-400.

12. Kobayashi, Y., Miyazawa, M., Araki, M., Kamei, A., Abe, K. \& Hiroi, T. (2015). Effects of Morus alba L.(mulberry) leaf in hyper-cholesterolemic mice on suppression of cholesterol synthesis. Journal of Pharmacognosy and Natural Products, 2, 113-114.

13. Athira, K., Madhana, R.M. \& Lahkar, M. (2016). Flavonoids the emerging dietary supplement against Crisplatin induced nephrotoxicity. Chemico- Biological Interactions, 248, 18-20.

14. Naveed, U., Mir, A.K., Salimullah, K., Habib, A., Afzal, H.A. \& Taous, K. (2016). Nephroprotective potential of Morus alba, a prospective experimental study on animal models. Journal of Pharmaceutical Biology, 54,3,530-535.

15. Arise, R.O., Bankole, S.I., Aboyewa, J.A. \& Bobbo, R. (2017). Antidiabetic and safety properties of ethanolic leaf extracts of Corchorus olitorius in alloxan -induced diabetic rats. Diabetes Food Plan, 4,60-69.

16. Naveed, U., Mir, A.K., Salimullah, K., Habib, A., Afzal, H.A. \& Taous, K. (2016). Nephroprotective potential of Morus alba, a prospective experimental study on animal models. Journal of Pharmaceutical Biology, 54,3,530-535.

17. Rahimi-Madiseh, M., Naimi, A., Heydarian, E. \& Rafieian-Kopaei, M. (2017). Renal biochemical and histopathological alterations of diabetic rats under treatment with hydro alcoholic Morus nigra extract. Journal of Renal Injurj Prevention,6, 1-3.

18. Cho,N.H., Kirigia,J., Mbanya, J.C., Ogurstova, K.,Guariaguata,L. \& Rathman, W.(2017). International Diabetes Federation, Diabetes Atlas, Eighth Edition.pp 12-14. 